\title{
EDITORIAL
}

\section{The imperfect beauty}

\author{
Francesco Velardi ${ }^{1}$ Annalisa Cogliandro ${ }^{2,3} \cdot$ Paolo Persichetti $^{2,3}$ (1)
}

Received: 21 May 2021 / Accepted: 31 May 2021 / Published online: 8 July 2021

(c) The Author(s), under exclusive licence to Springer-Verlag GmbH Germany, part of Springer Nature 2021

The evaluation of the surrounding world, either existing in the realm of materiality or in the ideal universe of abstract imagination, the perception of ourselves and the others, the emotional quality of our conversations, our mating preferences, and even our reproductive decisions, is energetically oriented by physical appearance and beauty, as pervasive and powerful agents in the human society. The recent emphasis on platform communications and image sharing produced an increased interest in idealized physical looks. Automatic analysis and machine-learning algorithms have been developed for the digital enhancement of human physical attractiveness, as well as for other application areas, such as platform image sharing, computer-assisted search of partners in online dating services, animation, advertising, and computer games [1]. Rather than being just a joyful entertainment, in the contemporary social context, the struggle for "beauty" became an obsessive task, as the ongoing tremendous demand for cosmetic surgery stands for. Indeed, the perception of beauty (referred to the real world, the artistic production, let alone the human body and face) has long been debated amongst philosophers, artists, psychologists, neurologists, and anthropologists. One of the topics in question is whether the perception of beauty is a social, cultural, and learned concept or it is, rather, hard-wired into our brain from birth. Although beautiful persons are often consistently recognized per se, they do not necessarily share common features. Moreover, there is no general agreement yet, on which facial/bodily/vocal qualities and characteristics appear to make a human being appealing and should discriminate between beautiful and normal persons. The

Paolo Persichetti

EJPS@unicampus.it

Neurosurgeon - Casa di Cura Pio XI, Rome, Italy

2 Plastic and Reconstructive Surgery Department, Campus Bio-Medico University of Rome, Via Alvaro del Portillo 200, 00128 Rome, Italy

3 Research Group "To Be and To Appear: Objective Indication To Plastic Surgery" of Campus Bio-Medico University of Rome, Rome, Italy truth of the matter is that no consensus has been reached in almost any field, as we are still frantically in search for even a definition for beauty. If we were to conceptually inquire on art and aesthetic experience, we should explore many aspects of beauty. What is beauty? What do we mean when we say "beautiful" about something? Beauty is appreciated following the same canons and rules when it is perceived looking at or listening to someone or something? Are the characteristics of beauty referred to the material world different from those we perceive when referring to humanity? May all the conceived beauties pertain to the same realm, as the final product of a common brain process? Most of the Western as well as non-Western aesthetic theories share the same concept of beauty. Accordingly, beauty is conceived of as something harmonious, as an organic whole, as a "gestalt" rather than a mere addiction of separate parts and, hence, as an object of pleasure. It is to say that, according to "philosophical aesthetics", there exists a universally valid concept of beauty as there are respective criteria [2].

Should this be the case, what are they? May, at least some of these characteristics, be considered for a universally valid aesthetic judgment on "beauty"?

\section{Beauty, perfect and divine}

At the beginning of times, humanity, tenaciously motivated to comprehend the multifaceted aspects of reality, harmonized and simplified the observed phenomena arranging them in groups of "supreme values" as goodness, beauty, and truth.

Ancient philosophers believed that there is a timeless, aesthetic ideal concept of beauty based on proportions, symmetry, harmony, and geometry, independent of the observers themselves. They trusted that the divine laws were ruling both the immanent and the transcendent phenomena of the universe, including the human experiences. For the sake of idealism, only the most general, abstract, and immaterial idea of beauty is real and perfect beauty. As a consequence, from a logical point of view, objects were esteemed beautiful 
as far as they participated of the same idea of beauty. During the development of Greek aesthetics, there was a gradual shift in all spheres of art from symmetry to eurhythmic beauty, which did not need objective good proportions as far as it aroused pleasant feelings in the beholder. In this sense, when representing reality (therefore, even the face), it may appear beautiful even if slightly departing from perfect symmetry. The intellectualist stereotype of beauty conceived in classical antiquity persisted through the Middle Ages. Symmetry meant commensurability and was believed to constitute a canon of beauty in nature as in art. During the Italian Renaissance, the canons of beauty were still based on the classic Greek contributions.

The expressive approaches dominated the pictorial techniques addressed to the representation of human body. The artists, especially the scholars grown in the cultural medium of Florentine "bottegas," developed an authoritative skill in flawlessly portraying the human physiognomy. Their ability to reproduce the likeness, liveness, harmony, and proportion of the human face and body was mysteriously perfect. It was a short step for Leonardo da Vinci to assess, "We [painters] by our arts may be called the grandsons of God."

The representation of human physiognomy was focused in idealistic forms, neglecting the realistic evidence of anatomic features. Rather than rendering the actual aspect, the issues related to a generic typicity were privileged, thereby ruling out the likeness and the specificity of the portrayed sitter. This attitude determined that anomalies of the human figure, yet more of skull and face, were depicted in negligible occasions. The ultimate target of the artist was to reproduce the ideal symmetry characteristic of their subjects, rather than the imperfect natural objects, which were his models. His success was judged by the extent to which he was thought to have embodied this ideal symmetry in his work more perfectly than it was manifested in his models [3]. Far from the general attitude, Leonardo da Vinci subjectivized ratio, symmetry, and balance within the narrow limits of idiosyncrasy. "...A man may be well proportioned, and yet be tall or short, large or lean, or of a middle size... and whoever does not make great use of these varieties, which are all existing in Nature in its most perfect state, will produce figures as if cast in one and the same mould, which is highly reprehensible [4]. He expanded the realm of Beauty inside the bounds set by Humanity itself to such an intense and forceful vision that his "idea" of "anima" unequivocally alluded to a kind of individual "Self " ("As it seems to me, it should be considered that the anima that holds and governs each painted body is determined by our judgement, as it is the same anima that holds and governs our own soul") [5]. In this line, one should wonder how "the beauties of faces... (that)... in nature are never found replicated" manifest both in real world and in artworks.

\section{The imperfect beauty, laical, and bourgeoisie}

In the late fifteenth century, the historical transition in cultural and political orientation acted as the trigger for an allEuropean artistic evolution. New groups of patrons flowered, mainly in the Northern European countries. A search for a less idealistic naturalism was favored, with the complicity of the reformist sentiment harboring in those wealthy bourgeois and merchant classes. These new patrons had developed a strong self-confidence on their autonomy and individual identity, favoring paintings that included portraits of themselves, together with domestic scenes, children, and household goods. In commissioning their portrait, indeed, this new group of sponsors witnessed the interest in the individualized "au vive" depiction of their highly esteemed self. Northern European artists sought to show the world as it actually was [6] and depicted people in a way that made them look more human, with a greater complexity of emotions than had been previously seen, leading the way toward the realistic portraying of secular subjects. Their hallmark was the sense of reality, the intention to depict the unique appearance of a particular person. In such a way, each portrait was meant to express individual identity. The faithful likeness of the person's physical features, representing the sitter even in a real, unflattering way, became accepted as the portrait reproduced, as well, the picture of the sitter's individual "humanity," his identity, his inner life, character, virtues, and social role (the "anima" of Leonardo), qualities considered valuable "per se." Therefore, the objective representation of "imperfect" humanity and flawed settings gained dignity. Hence, portraying imperfect bodies or human deformities was no longer considered an oxymoron. The harmony of beauty was manifestly perceived even within imperfections and asymmetry. On their site, the painters of the Italian Renaissance were masters in representing the human body. They developed an authoritative skill in portraying the human physiognomy, to such an extent that their ability to reproduce the likeness, liveness, harmony, and proportion of the human face was mysteriously perfect. As a general keystone, however, the humanistic culture of Italian Renaissance continued to be focused on the representation of human physiognomy in idealistic forms, neglecting the realistic evidence of anatomic features. Rather than rendering the actual aspect, the issues related to a generic typicity were privileged, thereby ruling out the likeness and the specificity in portraying the sitter. This attitude determined the exiguous occasions in which anomalies of the human figure, yet more of skull and face, were depicted. Indeed, instances of pictorial "imperfections" were extremely rare for the major artists of the Italian Renaissance. Even Antonello da Messina, prominent ambassador of the Flemish style in Italy, 
never completely abandoned idealistic naturalism, which he, rather, masterly melted with aspects of the European descriptive realism. Nonetheless, around 1483/1484, Sandro Botticelli, who was considered "able to portrait faces that speech and breath, that seem alive but lack only the voice" [7], depicted the "Portrait of a Young Man" as his ideal of male beauty. The physiognomic craniofacial features of the unknown sitter, indeed, show the evidence of craniofacial anomalies consistent with the long-term outcome of an untreated mild-to-moderate non-synostotic craniofacial deformation (positional plagiocephaly) [8].

\section{The beauty of imperfection and the human rain}

Over time, it has been clear that beauty was understandable in scientific terms. "Scientific aesthetics" has properly been viewed as a domain of cognitive neuroscience [9], giving a substantial contribution to our basic understanding of some of the unique features of the human mind [10], and to practical issues of everyday life, as social judgment, mate choice, and consumers' decisions. Different schools of philosophical aesthetics agree that "universally valid aesthetic judgments" about beauty may be validated as a "gestalt" which causes pleasure in each beholder. These "universally valid rules" of how to aesthetically judge beauty are suggestive for fundamentals common to all human beings, as though there were some universally shared properties of the human perception [11]. This assumption opens the way to a new bulk of questions relative to the interrelation between the neurological bases of perception and the normative rules of aesthetic judgment. That is the relationship between the brain and mind. As for a synthetic overview, it could be acknowledged that attractive faces are processed by the brain as rewarding stimuli. Attractive (as well as unattractive) faces undergo processing within the brain's reward centers in the orbitofrontal-cortex (OFC) and striatum, with medial OFC being responsive to attractive faces, and lateral OFC being responsive to unattractive faces. In addition, the amygdala plays an important role in indexing the social relevance of stimuli in the external environment and, in line with this, appears consistently to be involved in evaluation of attractiveness, a trait that carries important social implications [12]. Although symmetry is considered as an indicator of the health of the individual during development in ideal conditions, fluctuating asymmetries of the face, instead, may better reflect the bearer's developmental stability [13]. Since the emotional expressions and facial mimicry are asymmetrical, Neuropsychology studies have documented that a mild facial asymmetry does undoubtedly have a positive effect on attractiveness judgment in certain situations. It has been recognized that certain facial features (including those contributing to attractiveness) in fact show directional asymmetry or antisymmetry. On the contrary, markedly reduced asymmetries may make the face appear "unnatural" and hence less attractive. Indeed, viewing perfectly symmetrical faces, especially in situations where emotions are displayed and verbal communication is conducted, may induce a feeling of unexplained oddity if usually observed asymmetries are not seen [4].

\section{Struggle for the unattainable beauty in the "social network" era}

Faces are central to human social interaction, and their aesthetic valuation has important and wide-ranging societal implications. Indeed, standards of beauty are vigorously effective in changing people's beliefs and quality of life. With the empowerment of the filmmaking "star system," beauty has become a potent blend of makeup, plastic surgery, muscle-sculpting, hair restoration, dental work, and dermatology [14]. Correspondingly, this plot has come as well to everyday life. Beauty is increasingly becoming a right. While once related mostly to objects, people are beginning to make a new demand: "the right to personal beauty: the right to live in and surrounded by beauty and, most of all, the right to be beautiful" [15]. From puberty onwards, young girls set up the use of cosmetics in order to look older and attract older boys, exaggerating sexual dimorphisms by increasing the contrasts of the brow and eye. Conversely, their mothers used cosmetics in order to disguise the flaws of age and maintain a youthful appearance [16]. Starting from very early ages, where one is still getting to know himself/ herself, to advanced ages where the negative effects of aging are part of life, individuals of all genders are vigorously affected by these "almost impossible to reach" standards. Today, social media is one of the most important factors contributing to the mental, emotional, physical, and spiritual health of an individual. Constantly portraying ideal beauty and body image comparisons, social media, more interactive than traditional media, show to be more compelling in the definition of "universal beauty standards." Indeed, thanks to platforms like YouTube and Instagram, makeup techniques like "contouring," "strobing," and "baking," which were once the well-guarded tricks of top makeup gurus, have now become accessible to all, democratizing beauty [17].

In contrast to traditional media, images found on social media are mostly user-generated and receive the most likes and comments. Many of these images are "selfies," created by social media users as a form of self-presentation: a way of showing who they are to others. Due to their centrality in the digital self-presentation strategies and self or other's perception of beauty, the most heavily engaged photos on social 
media are the images of "filtered" faces, in which the subject remove unwanted content from the original snap. The most avant-garde filters allow the user to virtually wear several of the most hyped lip colors and skin tones in a single swipe. Meanwhile, the face inadvertently bobs up considerably smoother, the lips plumped, the eyes widened and expertly lined, and the lashes lengthened to create a "perfect" picture. The "like" feature of most forms of social media is a powerful weapon on the interest of users, because it offers easily quantifiable and ostensibly unambiguous measure of positive audience feedback. However, viewing the attractive images of celebrities and friends on social media, and the appearance-related comments these images often receive, has the potential to negatively influence the body image of users. Young people struggle to achieve the targeted "universal beauty standards," keenly aware that they may not even get any better. The greatest majority of them posts, at the end, only the most attractive images of themselves. They perceive the communicated beauty ideals as unrealistic and unattainable, regarding their image on social media as biased and not corresponding with their appearance in reality. Indeed, as the body comes to stand as a primary attribute of personal identity, it is increasingly regarded as a symbol, with unlimited capacities for withstanding alterations and modifications. The body, no longer thought as decaying and potentially dysfunctional, likely to require healing measures, "is becoming a commodity not unlike a car, a refrigerator, a house, which can be continuously upgraded and modified in accordance with new interests and greater resources"' [18].

As a general outcome, people using the retouching filters on their social threads surrender, preferring to look like their retouched self. Most of the times, they would ponder distrustfully before showing off their filter-free face because it just doesn't look as good as it would once enhance. Therefore, the pursuit to be and appear as one really is has become a titanic effort. With filters, modification apps, and suggested "tweaks" so accessible, the "new selves" are fast becoming the new normal, with the line between natural and filtered beauty becoming increasingly blurred. By accepting this singular expression of beauty, there's an increasing danger that we are creating unrealistic beauty ideals. Over the past few years, surgeons have reported a disturbing number of people asking for procedures finalized at letting them look like they do in their social media filtered selfies. Mary Lynn Moran, $\mathrm{MD}$, facial plastic surgeon and president of the American Academy of Facial Plastic and Reconstructive Surgery (AAFPRS), exploring the top trends in facial plastic surgery in the release of the 2019 Annual Survey, revealed that the annual statistics continue to show significant increases in the social media category. "Clearly social media remains a huge incentive to have some work done with even more surgeons than last year reporting that patients are seeking cosmetic procedures to look better in selfies, Instagram,
Snapchat, Facebook Live and other social channels" (https:// apnews.com/press-release/pr-prnewswire/2fd0f769df827c9 9aeb279b89de8dd96). This trend has been termed "snapchat dysmorphia." Filtered images "blurring the line of reality and fantasy, could be triggering body dysmorphic disorder (BDD), a mental health condition where people become fixated on imagined defects in their appearance" [19].

At present, cosmetic surgery is passing through an identity crisis, as well as an acute ethical dilemma. A closer look from an ethical viewpoint makes clear that the surgeon who offers aesthetic interventions faces many serious ethical problems related with the identity of the surgeon as a healer [18]. Due to the apparent lack of a therapeutic purpose, for example, the intention to cure or rehabilitate, the concept of "health" should be enriched, in cosmetic surgery, with a stronger subjective dimension. It should be related to the individual perception and appreciation for the individual body. "The request for a surgical procedure aiming at the enhancement of the physical and psychological well-being of the patient cannot be labeled as a heteronomous evaluation. Rather, it should be considered as an expression of the full and conscious self-determination of the person. This is precisely the reason why cosmetic surgery may also be regarded as "the surgery of the soul" [20]. Should aesthetic surgery work according to market categories, it would run the risk of losing the view for the real need of patients and will be nothing else than a part of the beauty industry, not helping people, but just selling something. As the demand for aesthetic surgery has increased tremendously over the past few decades, it is essential to forecast the satisfaction possibly expected after an aesthetic surgical procedure and to determine the final results with objective and as precise as possible assessments [21]. Although aesthetic judgments are surely subjective, there should be no preclusion to objectively study the results of surgery, through rigorous scientific methods. With EBM extending its claims over all fields of medical sciences, Aesthetic Surgery should be included as well [22]. Indeed, the factual basis of a science of esthetics is not to settle whether some person is "objectively beautiful" but rather to determine whether some representative set, or sets of individuals, judge or experience him/her as beautiful or unattractive [23]. In fact the beauty should be considered as "an emerging reality composed by objective, subjective, and relational dimensions, and medicine has to take care only of the objective part of the problem. Beauty, in its complexity, deals with body morphology, which is objective and defines the exterior of the person. There are two realities, objective and subjective, which depend on this objective basis: the vision of the bodily "I" (how I see myself) and the perception of others about my body (how others see me). These two elements are subjective because they depend on subjective actions but, at the same time, they are also objective elements due to the subjects which are involved. "How 
I see myself" and "How I am seen" are crucial elements for everybody in relational life... This multidimensional vision of beauty, as a reality emerging from the three abovementioned dimensions, corresponds necessarily to a multidimensional way of solving the problems connected with beauty. That means active cooperation between the different specific competencies" [24].

"Plastic Surgery is not the "Medicine of Desires" and must remain within the epistemological frame of Scientific Medicine, maintaining the correct indications for aesthetic or reconstructive procedures as its objective reference. Thus a sensible work aiming at beauty cannot correspond to something that is not in tune with the truth of the person. Such a risk may be run in a society where "appearing" prevails over "being." In this context, Plastic Surgery must be founded on consistent ethical grounds so as not to be overwhelmed by the distorted demands of the market" [25].

Data availability Not applicable.

Code availability Not applicable.

\section{Declarations}

Ethics approval For this kind of article, formal consent from a local ethics committee is not required.

Consent to participate Not applicable.

Consent for publication Not applicable.

Conflict of interest The authors declare no competing interests.

\section{References}

1. Gunes H (2011) A survey of perception and computation of human beauty. Proceedings of the 2011 ACM Multimedia Conference and Co-Located Workshops - JHGBU 2011 Workshop, J HGBU'11. https://doi.org/10.1145/2072572. 2072580

2. Paul G (1988) Beauty and the aesthetic judgment of beauty. In: I. Rentschler, B. Herzberger and D. Epstein (Eds.) Beauty and the Brain - Biological Aspects of Aesthetics. Springer - Basel AG

3. Schulz K, Hayn-Leichsenring GU (2017) Face attractiveness versus artistic beauty in art portraits: a behavioural study. Front Psychol 8:2254. https://doi.org/10.3389/fpsyg.2017.02254

4. Kowner R (1996) Facial asymmetry and attractiveness judgment in developmental perspective. J Exp Psychol Hum Percept Perform 22(3):662-675

5. - Leonardo da Vinci, Trattato della Pittura (based on the Cod. Vaticano Urbinate 1270 - Author's translation)
6. Ridderbos B et al (2005) Early Netherlandish Paintings: rediscovery, reception and research. Amsterdam University Press, Amsterdam

7. Berenson B (1960) The passionate sightseer The diaries: 1947 to 1956. Simon and Shuster, New York

8. Velardi F (2019) Cranio-facial deformity in the Botticelli's "Portrait of a Young Man" (NG626). J Pediatr Neonat Individual Med 8(2):e080226. https://doi.org/10.7363/080226

9. Huston JP, Nadal M, Mora F, Agnati LF, Cela Conde CJ (Eds) (2015) Art, aesthetics and the brain. Oxford University Press

10. Kandel ER (2012) The age of insight. The quest to understand the unconscious in art, mind, and brain, from Vienna 1900 to the present. Random House, New York

11. Paul G (1988) Philosophical theories of beauty and scientific research on the brain. In: Rentschler I, Herzberger B, Epstein D (eds) Beauty and the brain - biological aspects of aesthetics. Springer, Basel

12. Hampshire A et al (2012) Dissociable roles for lateral orbitofrontal cortex and lateral prefrontal cortex during preference driven reversal learning. Neuroimage 59:4102-4112

13. Stephen I, Wei TK (2015) Healthy body, healthy face? Evolutionary approaches to attractiveness perception. In: Haque S, Sheppard E (eds) Culture and cognition: a collection of critical essays. Peter Lang, Bern

14. Dickey J (2014) Everyone is altered. The secret Hollywood procedure that has fooled us for year. https://mashable.com/2014/12/01/holly wood-secret-beauty-procedure/?europe=true (Last visited March 2021)

15. Béry A, Delprat L (2014) Beauty and esthetics: juridical approach. Orthod Fr 85(1):133-137

16. Wood L. Perceptions of female beauty in the 20th century Retrieved on March 2021 from https://barneygrant.tripod.com/perceptions.htm

17. Blessing Omakwu https://www.blessingomakwu.com/blog/beaut yinthesocialmediaera - September 29, 2018

18 Bishara S et al (2008) Aesthetic/cosmetic surgery and ethical challenges. Aesth Plast Surg 32:829-839

19. Rajanala S et al (2018) Selfies - living in the era of filtered photographs JAMA. Facial Plast Surg 20(6):443-444

20. Barone M, Cogliandro A, Persichetti P (2017) Ethics and plastic surgery/what is plastic surgery? Arch Plast Surg 44:90-92

21 Atiyeh BS, Hayek SN (2008) Numeric expression of aesthetics and beauty. Aesthetic Plast Surg 32(2):209-16 (discussion 217-9)

22. Chung KC et al (2009) Introducing evidence-based medicine to plastic and reconstructive surgery. Plast Reconstr Surg 123:1385-1389

23. Ben-Nun L (2016) Beauty of humans. Ben-Gurion University of Negev Retrieved on March 2021 from https://www.researchgate. net/publication/288944310

24. Tambone $\mathrm{V}$ et al (2015) How you become who you are: a new concept of beauty for plastic surgery. Arch Plast Surg 42(5):517-520

25. Persichetti P et al (2004) About beauty Plast Reconstruct Surg 14(1):270-271

Publisher's note Springer Nature remains neutral with regard to jurisdictional claims in published maps and institutional affiliations. 